\title{
Vorwort der Herausgeber
}

\author{
Timo Schmid • Markus Zwick
}

(C) Springer-Verlag GmbH Germany, part of Springer Nature 2018

Liebe Leserinnen und Leser,

ein wenig hat es gedauert bis wir Ihnen das Heft 1 des Bands 12 des AStA Wirtschafts- und Sozialstatistisches Archiv nun auch in Papierform anbieten können. In der Erstellung eines Heftes ergeben sich an unterschiedlichsten Stellen immer wieder Verzögerungen. Sollten Sie künftig auch schon vorher lesen möchten, was Sie dann absehbar auch als Heft erreichen wird, nutzen Sie bitte den Service ,Online First ' des Springer-Verlages. Unter https://www.springer.com/statistics/journal/ 11943 können Sie mit der redaktionellen Fertigstellung der Beiträge, die erscheinenden Artikel teilweise schon sehr frühzeitig lesen und nutzen. So waren einzelne Artikel der jetzigen Ausgabe schon im Januar und Februar dieses Jahres in der Rubrik ,Online First' zugänglich. Der Springer Verlag bietet darüber hinaus auch einen Alarm (alert), der Sie, so Sie eine E-Mail Adresse hinterlegen, über jeden neu online publizierten Artikel informiert.

Es ist uns eine große Freude, in dieser Ausgabe des AStA Wirtschafts- und Sozialstatistisches Archiv die siebte im Rahmen der Statistischen Woche gehaltene Lecture zu Ehren von Heinz Grohmann abdrucken zu können. Auf der Statistischen Woche 2017 in Rostock stellte Notburga Ott in Ihrem Vortrag den „Quasi-Standard“ im Bereich der Armutsmessung und Armutsbekämpfung in Frage (Ott 2018). Die kritische Prüfung/Evaluierung der Angemessenheit statistischer Methodik war stets

T. Schmid $(\bowtie)$

Institut für Statistik und Ökonometrie, Freie Universität Berlin, Berlin, Deutschland

E-Mail: timo.schmid@fu-berlin.de

\section{Zwick}

Institut für Forschung und Entwicklung in der Bundesstatistik, Statistisches Bundesamt, Wiesbaden, Deutschland

E-Mail: markus.zwick@destatis.de 
eine der Prämissen von Heinz Grohmann, dem diese Vorlesung/Vortrag gewidmet ist. Aktuelle Erhebungen wie der Household Finance and Consumption Survey (HFCS) oder wie die European Union Statistics on Income and Living Conditions (EUSILC) oder auch nationale Umfragen dienen der Wissenschaft als Datengrundlage für (statistische) Methodik zur Messung von Armuts- und Ungleichheitsindikatoren. Diese Verfahren basieren zumeist auf „Quasi-Standard“ Instrumenten wie der ,relativen Armutsquote“ oder der ,modifizierten OECD-Skala“. Die Autorin stellt in dem Beitrag diese Instrumente auf die Probe und erinnert an intensive Diskussionen in den 1980er zu Fragen nach normativen Basisentscheidungen und den angemessenen statistischen Instrumenten zur empirischen Messung von Armut und Ungleichheit.

Mit dem Thema der Armutsmessung in Deutschland beschäftigt sich auch der Artikel von Beste et al. (2018). Die Autoren untersuchen Einkommensverteilungen, mehrere Armutsmaße, Armutsrisikoquoten von Subpopulationen, Armutsfaktoren und soziale Auf- und Abstiege mit den beiden Haushaltsstichproben PASS (Panel Arbeitsmarkt und soziale Sicherung) und SOEP (sozio-oekonomisches Panel) und vergleichen die Ergebnisse. Motivation hinter der Untersuchung sind mögliche systematische Verzerrungen in Armutsanalysen, deren Ursachen in der Stichprobenziehung, Datenausfall (Unit- oder Item-Non-Response) oder Gewichtung liegen können. Als zusätzliche Referenz werden Armutsquoten auf der Grundlage des Mikrozensus herangezogen. Basierend auf den statistischen Analysen kommen die Autoren zu dem Schluss, dass signifikante Unterschiede in den Armutsmaßen basierend auf dem SOEP und PASS festgestellt werden können, die sich jedoch teilweise über den Beobachtungszeitraum auflösen. Im Weiteren werden im vorliegenden Aufsatz mögliche Gründe und weitere Analysen diskutiert und vorgestellt.

Mit Band 12 des AStA Wirtschafts- und Sozialstatistisches Archiv beginnen wir auch mehr oder weniger zwei neue Wege. Wie im letzten Heft schon angekündigt möchten wir das AStA Wirtschafts- und Sozialstatistisches Archiv auch dazu nutzen, Sie über inhaltliche und personelle Veränderungen an Hochschulen und Instituten mit statistischem Bezug sowie in den Statistischen Ämtern zu informieren. Wir beginnen dies in Kombination mit unserer schon etablierten Reihe der Interviews, die dankenswerterweise schon seit 2014 von Walter Krämer durchgeführt werden. Kurz vor Weihnachten interviewte Walter Krämer (2018) den neuen Präsidenten des Statistischen Bundesamtes (Destatis) Herrn Dr. Georg Thiel. Zum 1. November 2017 hat Herr Thiel die Leitung von Destatis von Herrn Dieter Sarreither übernommen und mit der von ihm initiierten ,Digitalen Agenda“ (Riede et al. 2018) schon viel frischen Wind in die Behörde getragen.

In dem Beitrag von Joachim Wagner (2018) stellt der Autor einen relativ neuen und bisher wenig genutzten Datenbestand der Forschungsdatenzentren der Statistischen Ämter des Bundes und der Länder vor. Mit den Einzeldaten der Außenhandelsstatistik (Transaction data for German exports and imports), die in verschiedenen Projektkonstellationen um weitere Unternehmensdaten erweitert wurden, konnten schon eine Reihe neuer wissenschaftliche Erkenntnisse gewonnen werden. Das Potential der Daten ist aber weit größer als bisher erschlossen. Es verwundert ein wenig, dass diese Daten in der empirischen Wirtschaftsforschung bisher doch recht wenig Anwendung gefunden haben. Es mag für den einen oder anderen Leser verwunderlich erscheinen, dass die im Beitrag zitierte Literatur häufig vom Autor selber 
stammt. Dies liegt aber vor allem daran, dass die Mikrodaten der Außenhandelsstatistik bisher außerhalb der zitierten Anwendungen nicht genutzt wurden. An den Ergebnissen, die diese Daten liefern kann es kaum liegen. Wie die zitierte Literatur zeigt, haben die Ergebnisse Eingang in sehr etablierte wissenschaftliche Journale gefunden.

Wir möchten in der Zukunft vermehrt den Raum im AStA Wirtschafts- und Sozialstatistisches Archiv nutzen, um bisher nicht gehobene aber verfügbare ,Datenschätze“ in der Anwendung und im Rahmen von Übersichtsartikeln vorzustellen. Der Beitrag von Joachim Wagner stellt hier den Anfang dar.

Nun wünschen wir Ihnen, liebe Leserinnen und Leser, viel Spaß bei der Lektüre der ersten diesjährigen Ausgabe von AStA Wirtschafts- und Sozialstatistisches Archiv.

Timo Schmid und Markus Zwick

\section{Literatur}

Beste J, Grabka M, Goebel J (2018) Armut in Deutschland - Ein Vergleich zwischen den beiden Haushaltspanelstudien SOEP und PASS. AStA Wirtsch Sozialstat Arch. https://doi.org/10.1007/s11943018-0221-4

Krämer W (2018) Interview mit dem Präsidenten des Statistischen Bundesamtes, Dr. Georg Thiel. AStA Wirtsch Sozialstat Arch. https://doi.org/10.1007/s11943-018-0218-z

Ott N (2018) Armutsmessung und Armutsbekämpfung: OECD-Skala und „Statistik“-Modell der Regelbedarfsberechnung auf die Probe gestellt. AStA Wirtsch Sozialstat Arch. https://doi.org/10.1007/ s11943-018-0222-3

Riede T, Tümmler T, Wondrak S (2018) Die Digitale Agenda des Statistischen Bundesamtes. Wirtsch Stat $1: 102-111$

Wagner J (2018) Germany's trade in goods. AStA Wirtsch Sozialstat Arch. https://doi.org/10.1007/s11943018-0219-y 